\title{
Impact of drilling fluid viscosity, velocity and hole inclination on cuttings transport in horizontal and highly deviated wells
}

\author{
Ali Piroozian • Issham Ismail • Zulkefli Yaacob • \\ Parham Babakhani · Ahmad Shamsul Izwan Ismail
}

Received: 21 April 2011 / Accepted: 7 July 2012/Published online: 8 August 2012

(C) The Author(s) 2012. This article is published with open access at Springerlink.com

\begin{abstract}
Deviation from vertical path makes drill cuttings to accumulate on the lower side of the wellbore that induces the formation of cuttings bed. Subsequently, relative problems occur while drilling. Excessive torque and drag, difficulties in running casing in hole and accomplishing good cementing jobs and mechanical pipe sticking are few of the classical examples of such problems. Therefore, a comprehensive understanding of influential parameters on hole cleaning seems to be essential. This paper indicates the results of an experimental study that was carried out to evaluate cuttings removal efficiency using three types of drilling fluid. The experimental works were conducted using a 17 -feet long opaque flow loop of 2 -in. diameter as the test section. For each test, the amount of cuttings transport performance (CTP) was determined from weight measurements. Viscosity was investigated together with other two influential parameters, namely fluid velocity and hole inclination under various flow conditions. It is shown that the increase in drilling fluid viscosity has improved CTP by approximately $8 \%$ at all angles provided the flow regime remained turbulent while velocity was kept constant. However, further increase of viscosity as flow regime was turning into transient or laminar flow, has lessened CTP by a total average of $12 \%$. It was also revealed that an incremental escalation in hole inclination from $60^{\circ}$ to $90^{\circ}$ has a positive effect on CTP, i.e., it will be improved up to $40 \%$. Drilling fluid velocity was found to
\end{abstract}

A. Piroozian $(\varangle)$ - I. Ismail · Z. Yaacob · P. Babakhani .

A. S. I. Ismail

Faculty of petroleum and renewable energy (FPREE),

University Technology Malaysia (UTM),

81310 UTM Skudai, Johor, Malaysia

e-mail: alipiroozian@yahoo.com have significant impact on CTP as it could attain maximum percentage of $98 \%$.

Keywords Cuttings removal efficiency -

Cuttings transport performance · Drill cuttings ·

Drilling fluid . Hole cleaning .

Fluid viscosity and fluid velocity

\section{Introduction}

The significance of drilling horizontal and highly deviated wells particularly in the near future has attracted many oil companies' interest. Economically speaking, it is not always sensible to use conventional vertical drilling in the case of offshore fields, and sometimes it is even out of reach. Thus, the best alternative is exploiting deviated wells to bypass most obstructions. However, this approach is not devoid of deficiency while drilling. Excessive torque/ drag, upright equivalent circulation densities, hindering drillpipe sliding which results in limiting the lateral reach of the well, lost circulation, barite sag, inefficient hole cleaning and frequent sticking are some of the engineering problems encountered while drilling horizontally. These obstacles directly and indirectly contribute many other problems (Cameron 2001).

All in all, the slightest nuance in the angle of deviation will lead to changing of the lifting power of the mud. As the direction of drilling is shifted from vertical to horizontal orientation, capacity of drilling fluid for carrying drill cuttings will reduce. This happens due to tendency of cuttings to lie down along the low sidewall of the annulus rather than being lifted out (Fadairo et al. 2009). The capacity of drilling fluid to carry the drill cuttings determines the quality performance of hole cleaning. As long as 
Fig. 1 Schematic diagram of the flow loop

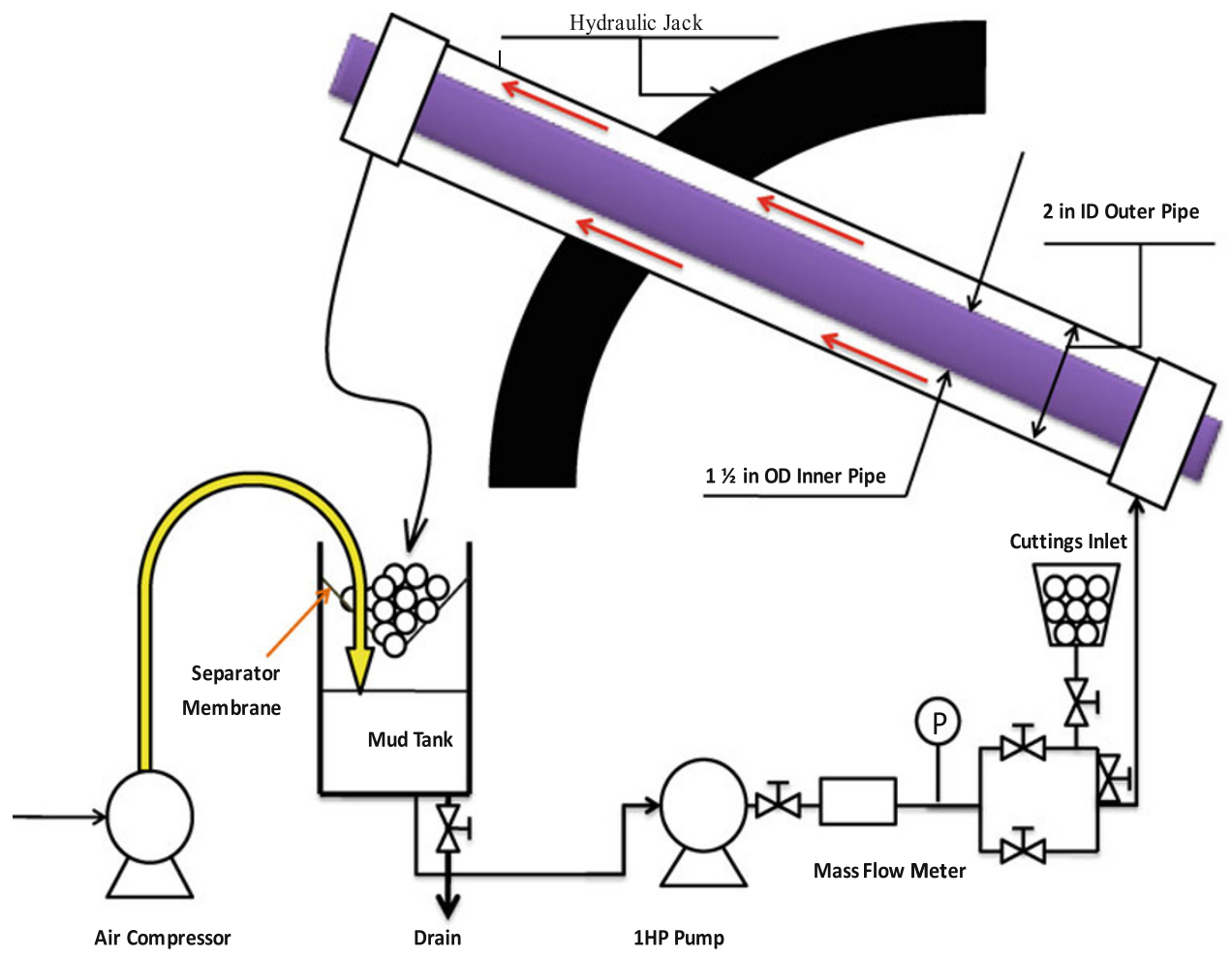

hole cleaning operation is done adequately, such engineering problems mentioned above can be avoided. Consequently, lower drilling time is required which reduces drilling expenditure significantly. It is beyond doubt that insufficient hole cleaning can reverse the operation outcome. Hence, a comprehensive understanding of hole cleaning is essential to prevent the unnecessary operational problems.

Most oilfield owners always work with drilling companies that can offer lower rig time. That is why penetration rate and the applied method go first. Besides, certain government restrictions should be taken into consideration before making any decision. Thus, all influential factors should be well examined beforehand. Strictly speaking, it is hard to turn a blind eye on the priority of cutting transportation in oil drilling operations especially after coupling with the potential well problems as highlighted in "Introduction", which has lead this study to focus on the issue.

Despite numerous studies on cuttings transport, little attention has been paid to the effect of drilling mud viscosity on CTP. Moreover, none of the researches have exactly defined flow conditions under which increasing the viscosity of drilling mud results in CTP enhancement and vice versa. Consequently, there exists a conflict regarding the effect of mud viscosity on CTP between researches fulfilled under laminar or transient flow and turbulent flow. Therefore, this research forwarded a new perspective to the subject using a filed scaled-down flow-loop apparatus. We have modeled 48 cases in this study to measure the CTP over four flow rates (i.e., 1.84, 2.21, 2.58 and $3.31 \mathrm{ft} / \mathrm{s}$ ) for four angles $\left(60^{\circ}, 70^{\circ}, 80^{\circ}, 90^{\circ}\right)$ with three fluid types. Eventually, the impact of each variable on CTP was discussed based on acquired data and information derived from published papers.

\section{Experimental work}

This study was carried on in Heavy Duty Lab, located in north part of UTM (University Technology Malaysia), to have sufficient vacant space to construct the designed rig of this research. In this research it is assumed that the weighting material, barite, added into the drilling fluid was well distributed during the circulation in the flow-loop apparatus. Another important assumption made was drill cuttings were homogenously dispersed throughout the annular space. Moreover, the entire length of PVC pipe used in the experiment work was considered to have uniform friction factor.

Preparation of each segment is briefly discussed as below:

\section{Flow-loop design and experimental setup}

With the aim to achieve the purpose of this study which was to examine the effect of three influential parameters on cuttings transport, namely fluid viscosity, hole inclination and fluid velocity, an experimental rig was built. The rig 
Table 1 Drilling fluid characteristics

\begin{tabular}{clll}
\hline $\begin{array}{l}\text { Mud } \\
\text { number }\end{array}$ & Composition & $\begin{array}{l}\text { Viscosity } \\
(\mathrm{cp})\end{array}$ & $\begin{array}{l}\text { Density } \\
(\mathrm{ppg})\end{array}$ \\
\hline $\begin{array}{c}\text { Mud } \\
\# 1\end{array}$ & Tap water & 1 & 8.345 \\
$\begin{array}{c}\text { Mud } \\
\# 2\end{array}$ & $\begin{array}{c}\text { Tap water }(350 \mathrm{ml})+\text { Bentonite } \\
(9 \mathrm{~g})+\text { Barite }(15 \mathrm{~g})\end{array}$ & 2.5 & 8.5 \\
$\begin{array}{c}\text { Mud } \\
\# 3\end{array}$ & $\begin{array}{c}\text { Tap water }(350 \mathrm{ml})+\text { Bentonite } \\
(9 \mathrm{~g})+\text { Barite }(15 \mathrm{~g})+\text { CMC } \\
(0.5 \mathrm{~g})\end{array}$ & 6 & 8.7 \\
& & & \\
\hline
\end{tabular}

has the potential to carry out all specified experiments in a low-pressure and low-temperature (ambient conditions) system (Fig. 1).

Field conditions had been considered in determining the size and the shape of the annulus flow section. For this study the section selected is planned to be drilled with $216 \mathrm{~mm}$ bit $(\approx 8.5 \mathrm{in}$.) while utilizing large-diameter drillpipe of $168 \mathrm{~mm}$ (6.5/8 in.). The test section was 17 feet long $(\approx 5 \mathrm{~m})$, consisting of an opaque PVC outer casing ( $2 \mathrm{in.}$ ID and $2^{1 / 2} \mathrm{in}$. OD) and a hollow steel inner drill pipe ( $1 \frac{1}{2}$ in. OD). A 1 H.P. centrifugal pump was used to circulate fluid into the flow loop. The cuttings were injected at the inlet of the annular test section where they merge with the test section.

Coarse sands taken from the Lotus Desaru Beach were selected as solid particles. They were sieved, washed, dried, and weighed, respectively. All particles had an irregular shape with average size of $1.70 \mathrm{~mm}$. After drying all sands, they were separated using a plastic bag into groups of $163 \mathrm{~g}$ each.

\section{Drilling mud}

Water-based drilling muds illustrated in Table 1 were prepared containing various amounts of bentonite and carboxymethyl cellulose (CMC) for this project. Stock suspension of bentonite was prepared by adding bentonite to tap water and shearing at a high rate for $6 \mathrm{~h}$. Stock solutions of the polymers were prepared by dissolving the polymers in tap water at a low shear rate and stirring continuously until clear solutions were obtained. The mud finally was mixed at a moderate shear rate with a mixer.

\section{Experimental procedure}

The first step to start the experiment was to prepare the cuttings. After cuttings were sieved and washed, it was time to dry it, weight it to $162 \mathrm{~g}(0.324 \mathrm{lb})$ and then placed in a plastic bag. The total amount of sieved cuttings which were prepared prior to each run exceeded $30 \mathrm{~kg}(66.2 \mathrm{lb})$. Each run consisted of four experiments and was dedicated for only one angle. All of these experiments were run
Experimental Procedure chart

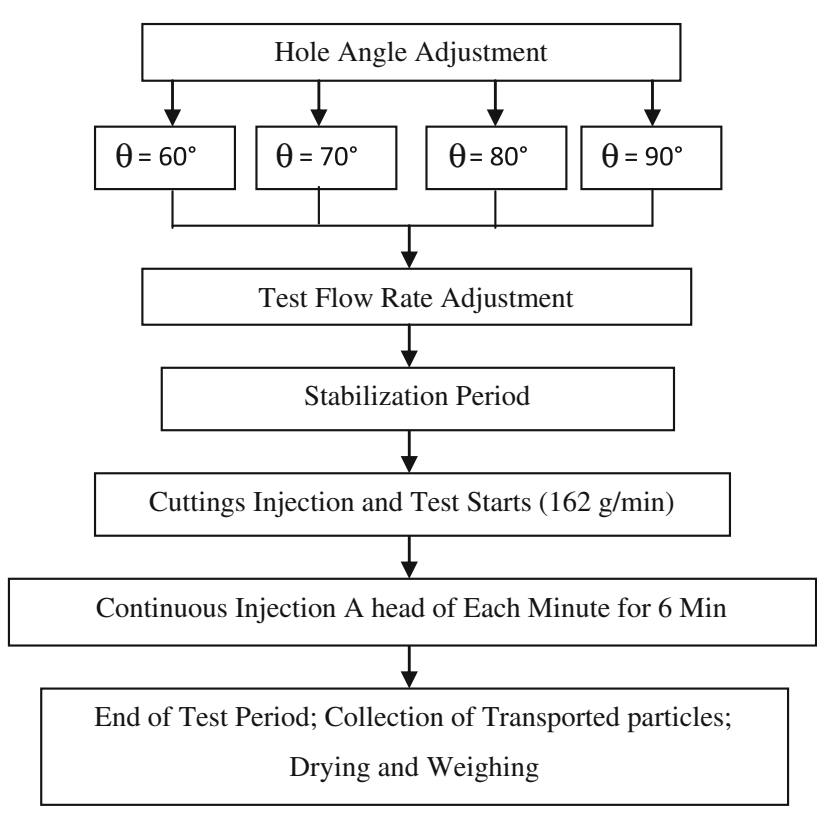

Fig. 2 Experimental procedure chart

without rotating the inner pipe for four flow rates (37.9, $45.4,53.0$, and $68.1 \mathrm{~L} / \mathrm{min}$ ). For the subsequent experiment, the same previous pattern was followed except using a new angle. This trend was repeated till all four experiments were fully implemented (see Fig. 2). After completion of all experiments and termination of first run, the second run was carried out with a new mud with a new viscosity (higher viscosity). To do so, CMC was used as another viscosifier besides bentonite.

Percentage of recovery can be calculated using the following equation:

Recovery $(\%)=\frac{\text { Final dried weight } \times 100}{\text { Intial dried weight }}$

\section{Results and discussion}

\section{Effect of fluid viscosity}

According to this study, increasing the plastic viscosity of the mud results in a remarkable increase in the amount of recovered cuttings. Surprisingly enough, the surplus amount of viscosity inverses the result. These phenomena are clearly seen in Fig. 3a-d.

As far as cuttings transport in highly deviated wells is concerned, researchers offer various ideas about the effect of viscosity on hole cleaning. Some researchers such as Zeidler (1972), Okrajni and Azar (1986), Pilehvari et al. (1999), Jawad (2002), Kelessidis et al. (2007) and Mohammadsalehi and Malekzadeh (2011) believe that 

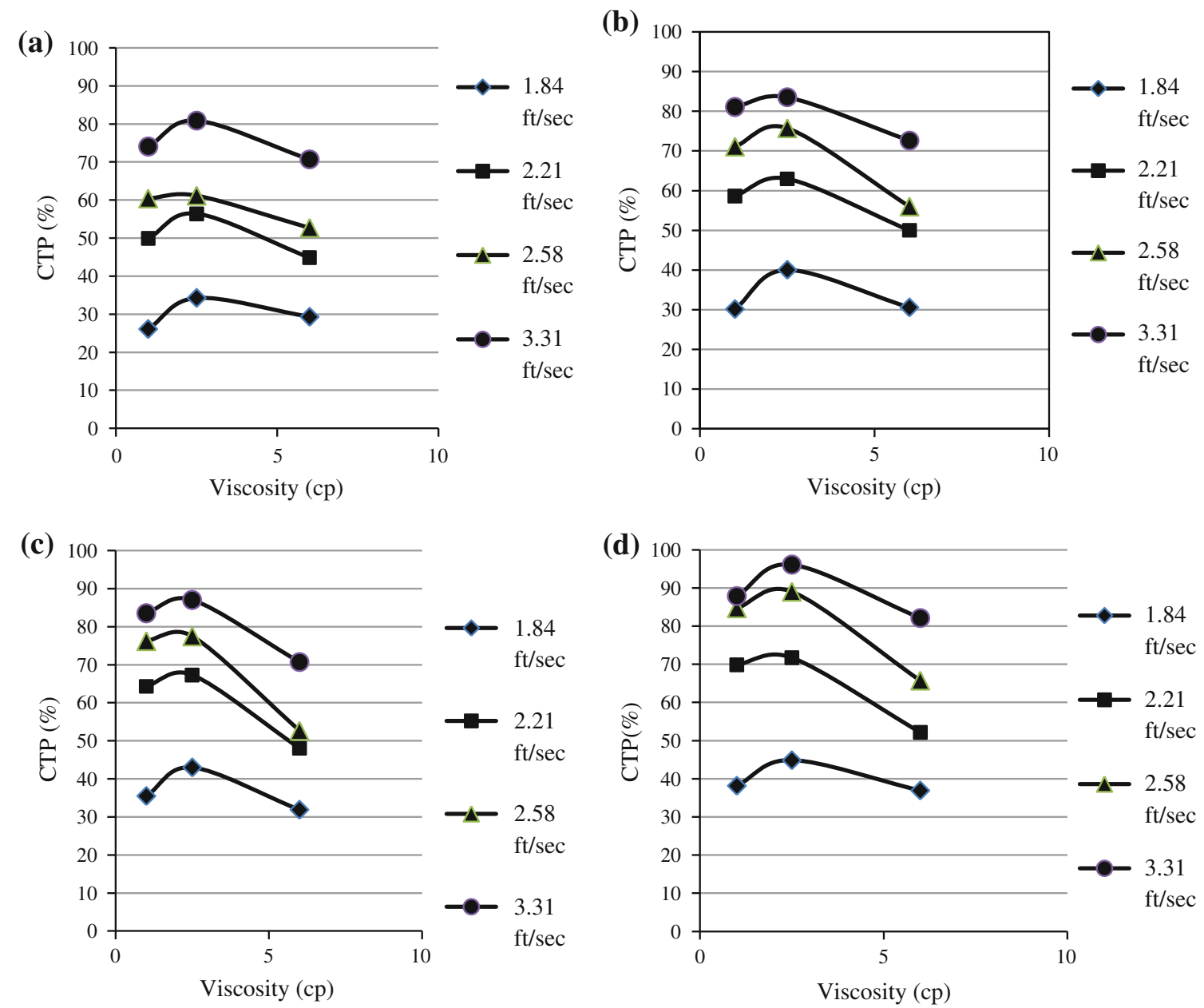

Fig. 3 Cuttings transport performance versus viscosity for four distinct hole inclinations from vertical, i.e. a $\theta=60^{\circ}$, b $70^{\circ}$, c $80^{\circ}$ and $\mathbf{d} 90^{\circ}$, with different flow velocities, i.e. $1.84,2.21,2.58,3.31 \mathrm{ft} / \mathrm{s}$

raising viscosity of the drilling fluid deteriorates hole cleaning, because type of flow regime changes from turbulent flow to laminar flow; and it has been proved that cuttings can be better displaced in turbulent flow than laminar flow. On the other hand, there are also some investigators, for instance, Ford et al. (1990), Iyoho and Takahashi (1993), Belavadi and Chukwu (1994), Shou (1999), Li et al. (2004) who claim that improvement in hole cleaning occurs as viscosity increases.

Figure $3 \mathrm{a}-\mathrm{d}$ are for $1.74 \mathrm{~mm}$ cuttings size and each figure is a representative of one specific angle (i.e., $60^{\circ}$, $70^{\circ}, 80^{\circ}$, or $90^{\circ}$ ). In all figures, cuttings transport performance (CTP) has been plotted versus viscosity. A line in each individual graph depicts a constant flow rate for the three distinctive types of drilling fluid with three different viscosities (1, 2.5, and $6 \mathrm{cp})$.

It is visible from Fig. $3 \mathrm{a}-\mathrm{d}$ that primary increasing viscosity from 1 to $2.5 \mathrm{cp}$ results in better CTP for all four different flow rates regardless of hole inclination. The effect of viscosity was, nevertheless, negative when the viscosity of the mud was intensified to $6 \mathrm{cp}$.

The best explanation for such a behavior of mud can be extracted from rheological laws defined for distinguishing various types of flow, namely laminar flow, transient flow, and turbulent flow. The type of flow of a fluid is characterized by the Reynolds number Re.

It is true to say that by increasing viscosity of the fluid at the same flow rate, the current flow regime tends to convert to laminar flow from turbulent flow. However, it should be noticed that converting a turbulent flow to laminar flow requires passing through a wide range of Reynolds number including transition zone. Thus, the author prepared a general graph which indicates how viscosity affects CTP by changing flow regime (Fig. 4).

It can be interpreted from Fig. 4 graph that CTP is improved by increasing viscosity while other factors such as velocity and hole inclination are kept constant. This happens until the flow is still turbulent, but once reaching 
Fig. 4 Effect of viscosity by changing the flow regime

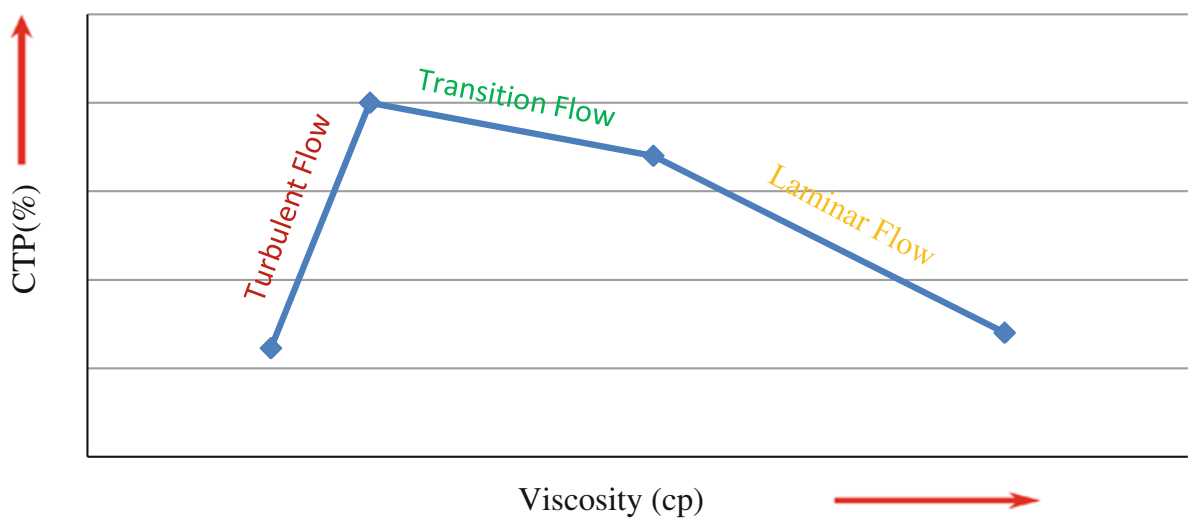

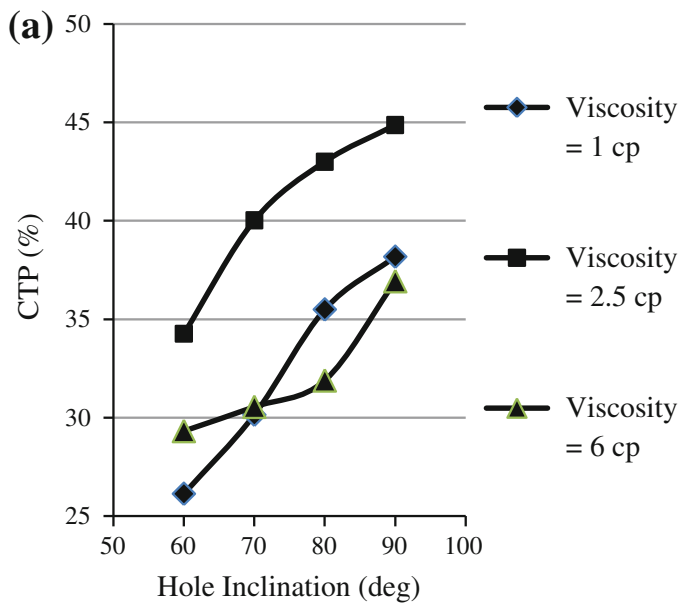

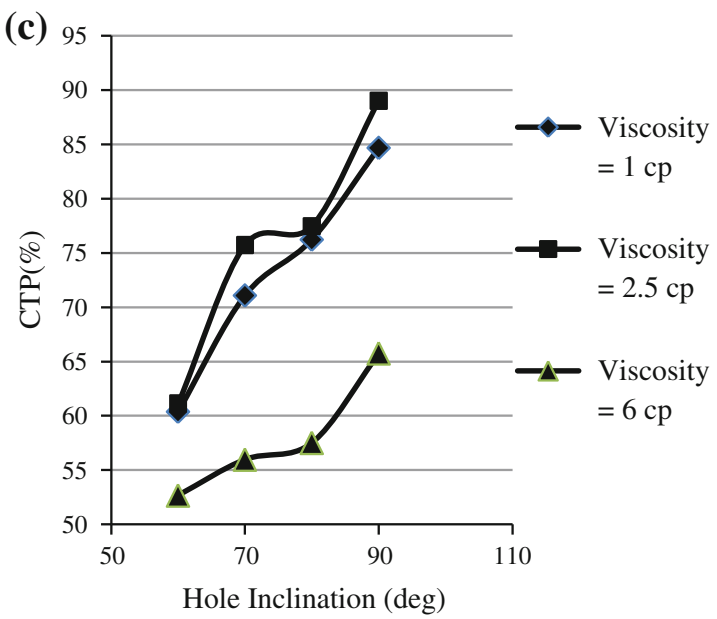

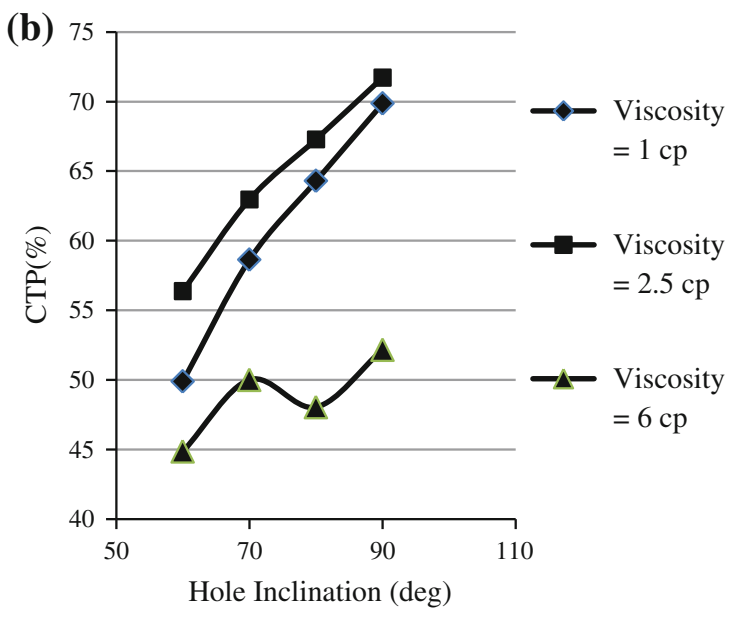

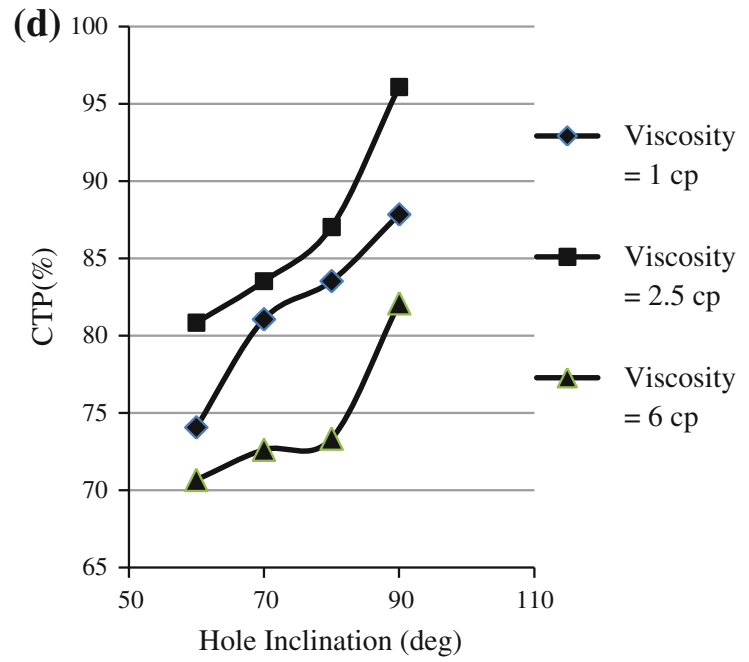

Fig. 5 Cuttings transport performance versus hole inclinations for four miscellaneous fluid velocities, i.e. a $V=1.84 \mathrm{ft} / \mathrm{s}, \mathbf{b} 2.21 \mathrm{ft} / \mathrm{s}, \mathbf{c} 2.58 \mathrm{ft} / \mathrm{s}$ and $\mathbf{d} 3.31 \mathrm{ft} / \mathrm{s}$, with different fluid viscosities, i.e. $1,2.5,6 \mathrm{cp}$

the transition zone CTP gradually decreases till the end of this region. Subsequently, laminar flow becomes transition flow by further increase of viscosity at the same condition. Among those three types of flow regimes, turbulent flow is the most desirable one followed by transition and laminar flows. The effect is prevailing at lower and higher velocities and for all hole inclinations. All these phenomena can be explained by the definition of each flow regime. As the flow is laminar, any laminar layer of the fluid is displaced, with respect to other laminar layers, in parallel to the direction of flow, and is moving at its individual speed. For flow through a cylindrical tube, the flow rate is 

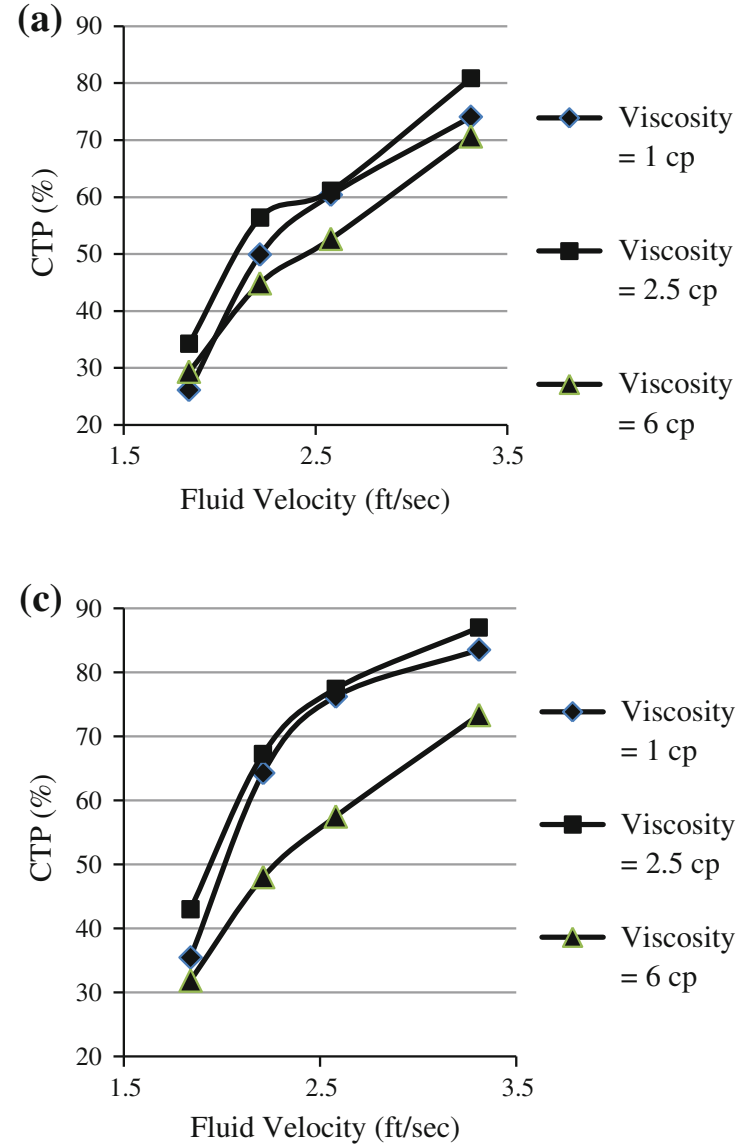
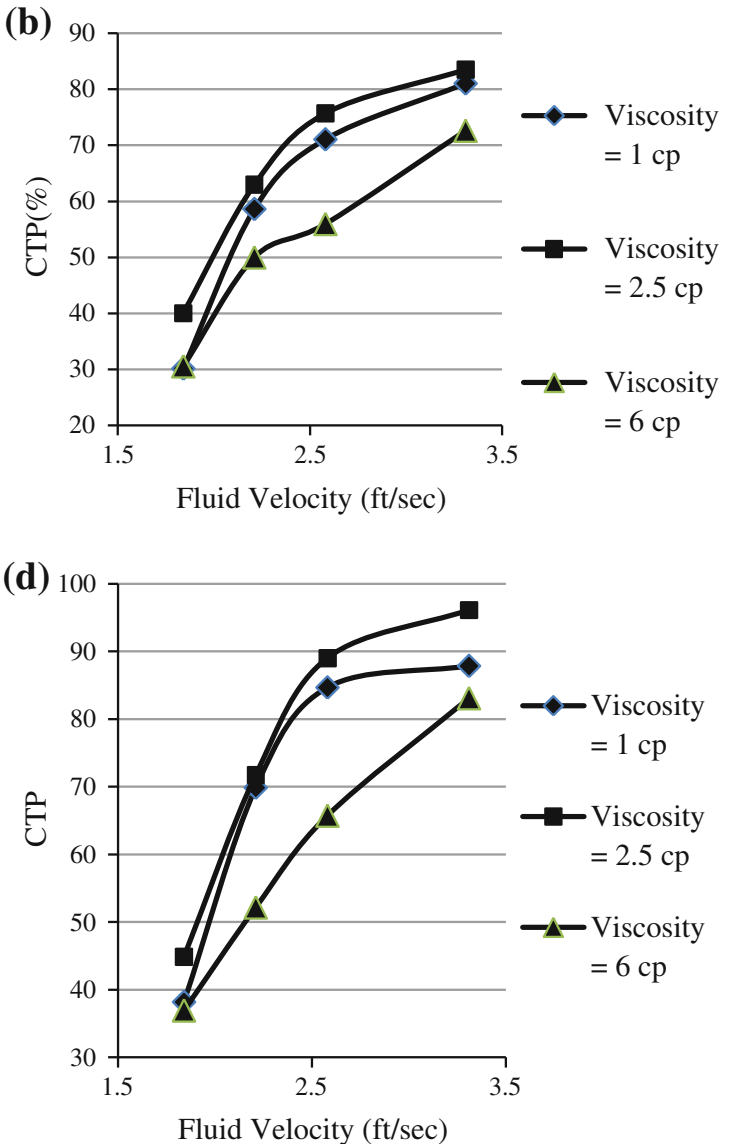

Fig. 6 Cuttings transport performance versus fluid velocity for four distinct hole inclinations from vertical, i.e. a $\theta=60^{\circ}$, b $70^{\circ}$, c $80^{\circ}$ and d $90^{\circ}$, with different fluid viscosities, i.e. $1,2.5$ and $6 \mathrm{cp}$

highest along the axis of the tube. At the tube wall it is zero throughout the volume of the fluid. However, turbulent flow consists of small eddies throughout the volume of the fluid, and this character of this type of flow produces more momentum force which gives better movement of cuttings of course.

The resistance of drilling fluid to flow is defined by the term called plastic viscosity which indicates the extent of physical and chemical interactions between fluids and solid particles applied into the mud. In general, any increment of solid content in drilling mud, such as barite and fluid loss materials, will result in higher plastic viscosity. The presence of shale particles in drilling fluid system when drilling through a shale zone will also increase the plastic viscosity. Strictly speaking, the higher plastic viscosity generates higher resistance in mud which in turns will affect cutting lifting performance. The situation may be worsened by the increase of ultra-fine drill solids in the drilling fluid which causes incremental trend of plastic viscosity at constant mud weight. This phenomenon explains how geology variations impacts on the cutting transport. Therefore, the size of drill cuttings as well as formation shale content should be put into consideration before designing the drilling fluid. In this research work, clean sand particles of $1.70 \mathrm{~mm}$ were used as the drill cuttings. Therefore, their effect on mud viscosity was negligible.

\section{Effect of hole inclination}

In the laboratory works it was observed that the angle of the well played an important role in transporting drill cuttings. This is the reason why hole cleaning is more challenging in directional wells as compared to vertical wells. Figure 5a-d clearly illustrate the effect of hole inclination on hole cleaning. In each curve, hole inclinations have been plotted versus CTP for one value of velocity and different liquid viscosities. For high angles, where a stationery cuttings bed can form, transport is via rolling mechanism. In intermediate angles, where a churning, moving cuttings bed can form, transport is via a lifting mechanism. At near-vertical angles, particle settling determines transport.

Lines in these curves indicate types of chosen mud in every run of the experiment. Rhombic markers belong to the mud with viscosity of $1 \mathrm{cp}$, square markers have a viscosity of $2.5 \mathrm{cp}$ and triangle markers have the highest 
viscosity by the value of $6 \mathrm{cp}$. The existing distance between lines also shows different types of mud with different rheological properties. Four angles had been selected for this study namely $60^{\circ}, 70^{\circ}, 80^{\circ}$, and $90^{\circ}$ from vertical. All lines give an upward trend by diverging from vertical, indicating that CTP has improved. Some researchers such as Okrajni and Azar (1986), Ford et al. (1990), and Bilgesu and Chukwu (2007), believed that if hole angle increases, their negative influences on cutting transports will increase as well. However, the author believes that it also has some positive effects especially when the flow is laminar. It is obvious that at higher degrees of inclination the tendency of downward cuttings bed sliding is more likely to occur which increases the hydraulic requirement for adequate hole cleaning. These beds moved at a lower speed along the annular space so that at the end of the test period, there may be some beds about to approach the outlet of the annulus section and if the test period has lasted for a few extra minutes there is a great chance for the nearby moving beds to leave the annular section. Thus, it is believed that a slight increase in hole inclination causes these large moving bed to reach the outlet before the end of the test period; thus a considerable increase in WRP and CTP.

\section{Effect of annular fluid velocity}

When the influence of velocity on hole cleaning was analyzed, it has been observed that the velocity has a positive contribution of removal of stationary bed. This is an interpretation taken form Fig. 6a-d. As it can be seen in all figures, the incremental increase in annular velocity brings a substantial increase in cuttings transport. The effect is more significant in higher hole inclinations and higher fluid viscosities.

Most portions of the injected cuttings were recovered by highest velocity of the drilling fluid and the most viscous mud. This fact also supports previous discussion about viscosity of mud in which increasing the viscosity can improve CTP if the flow regimes is still turbulent.

All in all, these results coincide well with vast majority of previous experimental work. Many researchers have already reported the effect of flow rate on hole cleaning, such that as the flow rate is increased, a reduction on cuttings bed area will be experienced.

\section{Conclusions}

This research study was aimed to more concentrate on the effect of viscosity on CTP, since there are some contradictions among previous studies about this issue. To do so, a simulated borehole was designed and constructed.
From all the influential parameters only three of them, i.e., hole inclination, fluid viscosity and velocity, were selected regarded to the objectives of this study. The important points discussed in this study are summarized below:

1. According to this study, increasing the plastic viscosity of the mud results in a remarkable raise in the amount of recovered cuttings. Surprisingly enough, the surplus amount of viscosity inverses the result. This phenomenon is explained well using a proposed graph which has been prepared by the author. From the graph it can be observe that CTP is improved by approximately $8 \%$ at all angles via increasing viscosity while other factors such as velocity and hole inclination are kept constant. This happens until the flow is still turbulent, but reaching the transition zone CTP gradually decreases, by a total average of $12 \%$, till the end of this region. Subsequently, laminar flow is substitute for transition flow by further increasing of viscosity at the same condition. Among those three types of flow regimes, turbulent flow is the most desirable one flowed by transition and laminar flows. The effect is prevailing at lower and higher velocities and for all hole inclinations.

2. During all experiments in this investigation it was observed that the angle of the annulus played an important role in transporting of drilled cuttings. This is the reason why hole cleaning is more challenging in directional wells rather than the vertical wells. Some researchers believe that if hole angle increases, their negative influences on cutting transports will increase as well. However, the operator of this study believes that it also has some positive effects especially when the flow is laminar. It is obvious that at higher degrees of inclination the tendency of downward cuttings bed sliding is more likely to occur which increase the hydraulic requirement for adequate hole cleaning. These beds moved at a lower speed along the annular space so that at the end of the test period, there may be some beds about to approach the outlet of the annulus section and if the test period has lasted for a few extra minutes there is a great chance for the nearby moving beds to leave the annular section. Thus, it is believed that a slight increase in hole inclination causes these large moving bed to reach the outlet before the end of the test period; thus a considerable increase in WRP and CTP up to $40 \%$.

3. When the influence of velocity on hole cleaning was analyzed, it has been observed that the velocity has a positive contribution of removal of stationary bed. The incremental increase in annular velocity brings a substantial increase in cuttings transport as it could attain maximum percentage of $98 \%$. The effect is 
more significant in higher hole inclinations and higher fluid viscosities. The most portions of injected cuttings were recovered by highest velocity of the drilling fluid and the most viscous mud. This fact also supports previous discussion about viscosity of mud in which increasing the viscosity can improve CTP if the flow regimes is still turbulent.

4. Even though the performance of fresh water and mud of $2.5 \mathrm{cp}$ seems quite alike in some figures particularly in Fig. 6, it was still imperative to use both to have different type of fluid-flow regimes under predetermined conditions of this study. However, it is apparent to mud engineers that fresh water could not be a rational alternative of mud selection for drilling any oil and/or gas wells because of difficulties caused by its usage such as clay swelling, dispersion of clay and other uninvited problems. Inadequate hole cleaning is a major problem in drilling operations and will contribute to various drilling-related problems, such as high rotary torque, stuck pipe, formation break down, slow rate of penetration and lose of circulation. This in turn will lead to higher drilling cost for remedial job, replacement of failed equipment as well as additional cost for the downtime due to installation job and increase in drilling running time. Strictly speaking, the better the efficiency of the cutting transports performance, the more is the drilling time saved and therefore the greater implicit reduction in expenditure.

Open Access This article is distributed under the terms of the Creative Commons Attribution License which permits any use, distribution, and reproduction in any medium, provided the original author(s) and the source are credited.

\section{References}

Belavadi MN, Chukwu GA (1994) Experimental study of the parameters affecting cutting transportation in a vertical wellbore annulus. Paper presented at the SPE Western Regional Meeting, Long Beach, California, 1 Jan 1994

Bilgesu MN, Chukwu GA (2007) Experimental study of the parameters affecting cuttings transport in a vertical wellbore annulus. SPE paper 27880 presented at the Western Regional Meeting held in Long Beach, California, 23-25 March

Cameron C (2001) Drilling fluids design and management for extended reach drilling. Paper presented at the SPE/IADC middle east drilling technology conference, Bahrain, 1 Jan 2001

Fadairo AS, Adekomaya O, Falode OA (2009) Effect of drilling cuttings transport on pressure drop in a flowing well. Paper presented at the SPE/IADC middle east drilling technology conference and exhibition, Manama, Bahrain, 1 Jan 2009

Ford JT, Peden JM, Oyeneyin MB, Gao E, Zarrough R (1990) Experimental investigation of drilled cuttings transport in inclined boreholes. Paper presented at the SPE annual technical conference and exhibition, New Orleans, Louisiana, 1 Jan 1990

Iyoho AW, Takahashi H (1993) Modeling unstable cuttings transport in horizontal, eccentric wellbores. Paper presented at SPE Eastern Regional Meeting, Pittsburgh, Pennsylvania, 1 Jan 1993

Jawad RH (2002) Carrying capacity design for directional wells. Paper presented at the IADC/SPE Asia Pacific drilling technology, Jakarta, Indonesia, 1 Jan 2002

Kelessidis VC, Bandelis GE, Li J (2007) Flow of dilute solid-liquid mixtures in horizontal concentric and eccentric annuli. (05). doi: 10.2118/07-05-06

Li Y, Bjorndalen N, Kuru EO (2004) Numerical modelling of cuttings transport in horizontal wells using conventional drilling fluids. Paper presented at the Canadian International Petroleum Conference, Calgary, Alberta, 1 Jan 2004

Mohammadsalehi M, Malekzadeh N (2011) Optimization of hole cleaning and cutting removal in vertical, deviated and horizontal wells. Paper presented at the SPE Asia Pacific oil and gas conference and exhibition, Jakarta, Indonesia, 1 Jan 2011

Okrajni S, Azar JJ (1986) The effects of mud rheology on annular hole cleaning in directional wells. SPE Drill Eng (08). doi:10.2118/14178-pa

Pilehvari AA, Azar JJ, Shirazi SA (1999) State-of-the-art cuttings transport in horizontal wellbores. SPE Drill Complet (09). doi: 10.2118/57716-pa

Shou G (1999) Solid-liquid flow system simulation and validation. Paper presented at the PSIG annual meeting, St. Louis, Missouri, 1 Jan 1999

Zeidler UH (1972) An experimental analysis of the transport of drilled particles. (02). doi:10.2118/3064-pa 